\title{
Numerical Simulation of Soil Water Movement under Subsurface Irrigation
}

\author{
Xinqiang Qin, Xianbao Duan, Lijun Su, Xiaoqin Shen, and Gang Hu \\ Department of Applied Mathematics, Xi'an University of Technology, Xian 710048, China \\ Correspondence should be addressed to Xinqiang Qin; xqqin2007@163.com
}

Received 30 April 2014; Revised 14 July 2014; Accepted 14 July 2014; Published 3 August 2014

Academic Editor: Kim M. Liew

Copyright (C) 2014 Xinqiang Qin et al. This is an open access article distributed under the Creative Commons Attribution License, which permits unrestricted use, distribution, and reproduction in any medium, provided the original work is properly cited.

\begin{abstract}
By constructing a radial basis function collocation method combined with a difference method, a two-dimensional mathematical model with boundary conditions of soil water movement under irrigation is proposed. The nonlinear term is dealt with a difference method and the equation is solved using an implicit scheme. In addition, the existence and uniqueness of the solution to the soil water movement equation are proven. Numerical results show that the proposed method has high precision and is easier to use than traditional methods. Moreover, the selection of parameter $c$ plays an important role in guaranteeing calculation precision. It lays the foundation for the numerical solutions to high-dimensional soil water movement equations.
\end{abstract}

\section{Introduction}

Between 1960 and 2000, numerical techniques such as boundary element and finite element methods have made spectacular advances in the computation of physical phenomena in engineering and sciences. Particularly, the last two decades have witnessed substantial efforts in developing a new class of numerical methods. These methods, using traditional methods that require certain underlying meshes such as the triangulation of a region for computation, were primarily developed for simulating low-dimensional application problems. However, designing relevant meshes is usually quite a difficult task for two-dimensional regions and might become impossible for higher-dimensional problems.

However, the triangulation process is too time consuming, even if a sophisticated mesh-generator is employed. In fact, for a given distribution of points, it is possible to achieve a mesh quickly, but this always requires a considerable number of iterations including manual interaction before reaching a satisfactory mesh.

Meshless methods were first introduced by Lucyt, and Gingold and Monaghan in 1977 [1, 2]. Further works, such as Nayroles et al. [3], Belytschko et al. [4], Schaback [5], Sukumar et al. [6], Wu [7], and Wendland [8, 9] (see books $[10,11])$, attempted to reduce or even eliminate the need for discretization of a domain or surface in the context of numerical solutions for boundary and initial value problems. These rapidly developed methods can not only reduce the large costs of labor, but also spare computational time compared to the finite element method, boundary element method, and other mesh-dependent methods.

The initial idea of meshless methods could date back to the smooth particle hydrodynamics (SPH) method for modeling astrophysical phenomena $[1,2]$. In 1992, Nayroles et al. proposed the diffuse element method (DEM) [3]. In 1994, Belytschko et al. improved the DEM and introduced the element-free Galerkin method (EFG) [4]. Since then, there has been a great deal of research into meshless methods. Meshless methods, such as the reproducing kernel particle method (RKPM) by Liu et al. [12], Hp-cloud method by Duarte and Oden [13], natural element method (NEM) by Sukumar et al. [6], partition of unity finite element method (PUFEM) by Babuška and Melenk [14], and meshless Galerkin method using radial basis functions (RBF) by Wendland [9], have also been described in literatures. The major differences between these meshless methods, all of which can be classified as Galerkin methods, come only from the techniques used for interpolating the trial functions. Even though no mesh is required in these methods for the interpolation of the trial or test functions and the solution 
variables, the use of shadow elements is unavoidable during the integration of the symmetric weak form. Therefore, these methods are not truly meshless.

The finite point method (FPM) introduced by Oñate et al. [15], which is based on the moving leastsquares and the collocation method, is a truly meshless method. Other truly meshless methods include the PCM [16], the Hp-meshless clouds [17], the LBIE [18], the LSC [19], and the WLSM [19] among others. In particular, in 1990, Kansa introduced the radial basis function (RBF) collocation method for solving elliptic, hyperbolic, and parabolic partial differential equations (PDEs) [20]. Later, the RBF was further developed by Schaback [5] and Fasshauer [21]. Hon et al. applied the RBF to the numerical computation of the variable normal equations, nonlinear Burgers equation, and shallow water wave equation [22]. In 1999, Rippa carried out work on selecting the correct shape parameter $c$ for the RBFs [23]. Another class of RBF was further developed by Wendland [8, 9], Wu [7], and Buhmann [24]. Zhang et al. have made more research on element-free $\mathrm{kp}$-Ritz method and applied it to solve different problems [25-29]. Therefore, the collocation-based meshless method has been an important meshless method in the current literature [30].

By creating a univariate basis function with an Euclidean norm, meshless methods are often naturally radically symmetric, and the high-dimensional problem can be turned into virtually one-dimensional one. Consequently, the study of the numerical solutions of PDEs through radial basis function interpolation has yielded a number of significant results.

1.1. The Problem and the Approach to Solve the Problem. The FEM has difficulty in remeshing and adaptive analysis. In contrast, meshless methods do not require a mesh to discretize the domain, and the approximate solution is constructed entirely with a set of scattered nodes [31]. However, meshless methods may lead to lower computational efficiency than FEM because more computational effort for the meshless interpolation and numerical integrations are required [32]. Hence, the improvement of the computational efficiency of meshless methods targeted at meshless interpolation and numerical integrations becomes an important issue. Other concerns or weaknesses of the existing meshless methods include difficulty in introducing the essential boundary conditions, a greater cost in evaluating the shape function derivatives, problems in handling discontinuities such as those due to heterogeneous material distributions, and the need for complicated node connectivity to ensure accurate results.

A truly meshless method, based on collocation with radial basis functions and radial basis functions are chosen to represent the solutions of PDEs, is the main focus of this paper. Moreover, the collocation-based meshless method is a truly meshless technique without mesh discretization. This method has the advantage of higher accuracy, convenient for computing, and has been successfully applied to numerical solutions of various PDEs. By using collocation with radial basis functions, the partition of the domain is not needed; hence the method can be applied to complex domains and

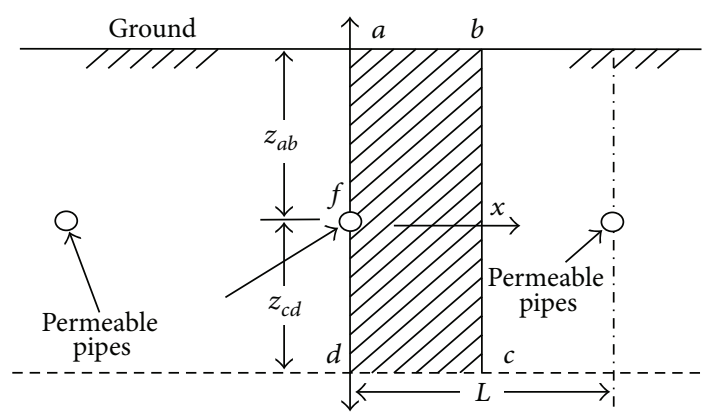

FIGURE 1: Schematic diagram of flow region.

overcomes some drawbacks of the traditional finite element method. It only needs to compute the shape functions and their derivatives, while the finite element method requires a calculation of the relevant integrals that often lowers the efficiency of computation significantly. The boundary conditions are relatively easier to be imposed without special manipulation. Finally, the way of obtaining the solutions to the PDEs is intuitive. From a practical point of view, this approach can achieve higher accuracy with more easily coded computer programs. Therefore, the collocation method with radial basis function interpolation can produce good results.

1.2. Mathematical Model of Soil Water Movement. Assume that permeable pipes in fields are parallel and have the same spacing and depth. Figure 1 shows a soil profile which is perpendicular to the permeable pipeline. Assuming that the water flow in the soil is negligible, then the problem considering irrigation can be solved as a plane problem. Obviously, in the case of Figure 1, it is sufficient to focus on soil water movement in the shaded part $a b c d$, namely, the flow region.

In the region of soil water flow, both perpendiculars $a d$ and $b c$ are symmetrical lines, where ad traverses through the center of a permeable pipe and $b c$ is equidistant from two adjacent permeable pipes. The symmetry means that the water flux along the normal direction of two lines equals zero. Line $a b$, the earth's surface, is the upper boundary of the region of soil water flow. The water flow which crosses line $a b$ is affected by the soil's conveyance capacity, which is in turn related to the surface soil transpiration and strength of rainfall. By ignoring the transpiration and rainfall effects, the water flux across $a b$ is zero. Line $d c$ is the lower boundary of the region of soil water flow but is deep enough so that water movement caused by irrigation cannot reach it.

Assume that, in the region of soil water flow, the soil water flow is continuous in time and space, obeying the law of mass conservation. Then we can develop mathematical models of the soil water movement based on the physical process through the area.

The mathematical model of 2D water movement in unsaturated soil can be established as

$$
\frac{\partial \theta}{\partial t}=\frac{\partial}{\partial x}\left(D(\theta) \frac{\partial \theta}{\partial x}\right)+\frac{\partial}{\partial z}\left(D(\theta) \frac{\partial \theta}{\partial z}\right)+\frac{\partial K(\theta)}{\partial z}
$$


where $\theta$ is the volumetric soil water content; $D(\theta)$ and $K(\theta)$ both are continuous functions which denote the diffusivity and hydraulic conductivity of the unsaturated soil flow respectively; $t$ is time and $z$ is the distance with the upward direction defined as positive.

The remainder of this paper is organized as follows. In Section 2, we introduce the construction of the meshless method based on the collocation method with radial basis functions, where the explicit schemes for the given equation are proposed and the existence and uniqueness of the solution to the method are proved. In Section 3, we give some applications and apply the method developed in this paper to examine the appropriateness and efficiency of the numerical solutions and to analyze the factors influencing the accuracy. We also compare our method to FEM. Section 4 comprises the conclusions and remarks.

\section{Construction of a Meshless Method Based on Collocation with Radial Basis Functions}

2.1. Radial Basis Functions. Radial basis functions (RBFs), also known as distance basis functions, are a type of functions with a basic variable $d_{j}\left(d_{j}=\left\|x-x_{j}\right\|\right)$. They are isotropic and simple in form and can be solved easily with numerical calculation. The method, which combines radial basis functions with collocation, has many advantages when solving the partial differential equation $[33,34]$. It has no correlation with the space dimensions and does not need any element or mesh for interpolation. Therefore, it is a truly meshless method. Currently, FEM [35] and FDM [36-38] are mostly used to solve soil moisture movement equations. With the development of meshless methods in recent years, many scholars now solve the soil water seepage problem using the finite volume method [39] and the RBF method [40]. The mathematical models of soil water movement are usually convection-diffusion equations, meaning that numerical oscillation occurs frequently when FDM or FEM is used. However, the radial basis function collocation method can solve many of these problems.

\subsection{Construction of Radial Basis Function Collocation Method.} The $2 \mathrm{D}$ soil water movement equation is

$$
\begin{gathered}
\frac{\partial \theta}{\partial t}=\frac{\partial}{\partial x}\left(D(\theta) \frac{\partial \theta}{\partial x}\right)+\frac{\partial}{\partial z}\left(D(\theta) \frac{\partial \theta}{\partial z}\right) \\
+\frac{\partial K(\theta)}{\partial z}, \quad(x, z) \in \Omega, \\
\theta(x, z, 0)=\theta_{0}, \quad(x, z) \in \Omega, \\
\theta(x, z, t)=\theta_{1}, \quad(x, z) \in \partial \Omega,
\end{gathered}
$$

where $\theta$ is the volumetric soil water content, $D(\theta)$ and $K(\theta)$ are the diffusivity and hydraulic conductivity of the unsaturated soil flow, respectively, $t$ is time, and $z$ is the distance with the upward direction defined as positive. $\theta_{0}$ is the initial water content, $\theta_{1}$ is the constant water content of ground under humid conditions, $\Omega$ is the seepage region, and $\partial \Omega$ is the boundary of $\Omega$.
Because the quadratic term in (2) is nonlinear, we cannot use the collocation method directly. Thus, we apply centered differences to deal with the nonlinear term in $\left(x_{i}, z_{j}\right)$ :

$$
\begin{aligned}
& \frac{\partial}{\partial x}\left[D(\theta) \frac{\partial \theta}{\partial x}\right]_{x=x_{i}} \\
& \approx \frac{1}{2 \Delta x}\left\{\left[D(\theta) \frac{\partial \theta}{\partial x}\right]_{x=x_{i+1}}-\left[D(\theta) \frac{\partial \theta}{\partial x}\right]_{x=x_{i-1}}\right\} \\
& =\frac{1}{2 \Delta x}\left[D\left(\theta_{i+1, j}\right)\left(\frac{\partial \theta_{i+1, j}}{\partial x}\right)_{x=x_{i+1}}\right. \\
& \left.-D\left(\theta_{i-1, j}\right)\left(\frac{\partial \theta_{i-1, j}}{\partial x}\right)_{x=x_{i-1}}\right] \\
& \frac{\partial}{\partial z}\left[D(\theta) \frac{\partial \theta}{\partial z}\right]_{z=z_{j}} \\
& \approx \frac{1}{2 \Delta z}\left\{\left[D(\theta) \frac{\partial \theta}{\partial z}\right]_{z=z_{j+1}}-\left[D(\theta) \frac{\partial \theta}{\partial z}\right]_{z=z_{j-1}}\right\} \\
& =\frac{1}{2 \Delta z}\left[D\left(\theta_{i, j+1}\right)\left(\frac{\partial \theta_{i, j+1}}{\partial z}\right)_{z=z_{j+1}}\right. \\
& \left.-D\left(\theta_{i, j-1}\right)\left(\frac{\partial \theta_{i, j-1}}{\partial z}\right)_{z=z_{j-1}}\right]
\end{aligned}
$$

where $\Delta x$ and $\Delta z$ are spatial intervals, respectively. $\left(x_{i}, z_{j}\right)$ are boundary points when $i, j=1, N$, are inner points when $i, j=$ $2,3, \ldots, N-1$.

Discretizing the left side of (2) with forward differences gives

$$
\left(\frac{\partial \theta}{\partial t}\right)_{\left(x_{i}, z_{j}\right)}^{t=t^{n+1}} \approx\left(\frac{\theta^{n+1}-\theta^{n}}{\Delta t}\right)_{\left(x_{i}, z_{j}\right)}=\frac{\theta_{i, j}^{n+1}-\theta_{i, j}^{n}}{\Delta t} .
$$

Discretizing the third term in the right side of (2) with central differences gives

$$
\left(\frac{\partial K(\theta)}{\partial z}\right)_{\left(x_{i}, z_{j}\right)}^{t=t^{n+1}} \approx \frac{K\left(\theta_{i, j+1}^{n+1}\right)-K\left(\theta_{i, j-1}^{n}\right)}{2 \Delta z}
$$

Let function $\tilde{\theta}\left(X, t^{n}\right)$ be an approximation of $\theta\left(X, t^{n}\right)$ :

$$
\tilde{\theta}\left(X, t^{n}\right)=\sum_{i=1}^{N_{I}} \alpha_{i}^{n} \varphi\left(\left\|X-I_{i}\right\|\right)+\sum_{i=1}^{N_{b}} \beta_{i}^{n} \varphi\left(\left\|X-B_{i}\right\|\right)
$$

where $X=(x, z), I_{i} \in \Omega$, and $B \in \partial \Omega . N_{I}$ is the number of the nodes in the region and $N_{b}$ is the number of the nodes on the border. 
Applying collocation method, (6) should satisfy the differential equation for the region $\Omega$ in (7) and boundary conditions on the borders $\partial \Omega$ in (8). That is,

$$
\begin{gathered}
\sum_{i=1}^{N_{I}} \alpha_{i}^{n}\left(\varphi\left(\left\|I_{j}-I_{i}\right\|\right)-\frac{d}{d x}\left(D\left(\theta^{n}\left(I_{j}\right)\right) \frac{d \varphi\left(\left\|I_{j}-I_{i}\right\|\right)}{d x}\right)\right. \\
\left.-\frac{d}{d z}\left(D\left(\theta^{n}\left(I_{j}\right)\right) \frac{d \varphi\left(\left\|I_{j}-I_{i}\right\|\right)}{d z}\right)\right) \\
+\sum_{i=1}^{N_{b}} \beta_{i}^{n}\left(\varphi\left(\left\|I_{j}-B_{i}\right\|\right)\right. \\
-\frac{d}{d x}\left(D\left(\theta^{n}\left(I_{j}\right)\right) \frac{d \varphi\left(\left\|I_{j}-B_{i}\right\|\right)}{d x}\right) \\
\left.-\frac{d}{d z}\left(D\left(\theta^{n}\left(I_{j}\right)\right) \frac{d \varphi\left(\left\|I_{j}-B_{i}\right\|\right)}{d z}\right)\right) \\
=\theta_{i, j}^{n}+\frac{\partial K\left(\theta^{n}\left(I_{j}\right)\right)}{\partial z}, \quad j=1,2, \ldots, N_{I},
\end{gathered}
$$

$$
\begin{gathered}
\sum_{i=1}^{N_{I}} \alpha_{i}^{n} \varphi\left(\left\|B_{j}-I_{i}\right\|\right)+\sum_{i=1}^{N_{b}} \beta_{i}^{n} \varphi\left(\left\|B_{j}-B_{i}\right\|\right) \\
\quad=\theta_{1}^{n}\left(B_{j}\right) . \quad j=1,2, \ldots, N_{b} .
\end{gathered}
$$

Let

$$
\begin{aligned}
& \psi(\|X-I\|)= \varphi(\|X-I\|) \\
&-\frac{d}{d x}\left(D(\theta(X))\left(\frac{d \varphi(\|X-I\|)}{d x}\right)\right) \\
&-\frac{d}{d z}\left(D(\theta(X))\left(\frac{d \varphi(\|X-I\|)}{d z}\right)\right) \\
& f^{n}\left(I_{j}\right)=\theta^{n-1}\left(I_{j}\right)+\frac{\partial K\left(\theta^{n}\left(I_{j}\right)\right)}{\partial z} .
\end{aligned}
$$

Then (7) and (8) can be expressed with the following matrix equation:

$$
\mathrm{HU}=\mathrm{F},
$$

2.3. The Existence and Uniqueness of the Solution. Let

$$
\begin{gathered}
\mathbf{A}_{1}=\left[\begin{array}{ccc}
\psi(0) & \cdots & \psi\left(\left\|I_{1}-I_{N_{i}}\right\|\right) \\
\vdots & \ddots & \vdots \\
\psi\left(\left\|I_{N_{i}}-I_{1}\right\|\right) & \cdots & \psi(0)
\end{array}\right], \\
\mathbf{A}_{2}=\left[\begin{array}{ccc}
\psi\left(\left\|I_{1}-B_{1}\right\|\right) & \cdots & \psi\left(\left\|I_{1}-B_{N_{b}}\right\|\right) \\
\vdots & \ddots & \vdots \\
\psi\left(\left\|I_{N_{i}}-B_{1}\right\|\right) & \cdots & \psi\left(\left\|I_{N_{i}}-B_{N_{b}}\right\|\right)
\end{array}\right], \\
\mathbf{A}_{3}=\left[\begin{array}{ccc}
\varphi\left(\left\|B_{1}-I_{1}\right\|\right) & \cdots & \varphi\left(\left\|B_{1}-I_{N_{i}}\right\|\right) \\
\vdots & \ddots & \vdots \\
\varphi\left(\left\|B_{N_{b}}-I_{1}\right\|\right) & \cdots & \varphi\left(\left\|B_{N_{b}}-I_{N_{i}}\right\|\right)
\end{array}\right],
\end{gathered}
$$

(7) where

$$
\begin{aligned}
\mathbf{H} & =\left[\begin{array}{cccccc}
\psi(0) & \cdots & \psi\left(\left\|I_{1}-I_{N_{i}}\right\|\right) & \psi\left(\left\|I_{1}-B_{1}\right\|\right) & \cdots & \psi\left(\left\|I_{1}-B_{N_{b}}\right\|\right) \\
\vdots & \vdots & \vdots & \vdots & \vdots & \vdots \\
\psi\left(\left\|I_{N_{i}}-I_{1}\right\|\right) & \cdots & \psi(0) & \psi\left(\left\|I_{N_{i}}-B_{1}\right\|\right) & \cdots & \psi\left(\left\|I_{N_{i}}-B_{N_{b}}\right\|\right) \\
\varphi\left(\left\|B_{1}-I_{1}\right\|\right) & \cdots & \varphi\left(\left\|B_{1}-I_{N_{i}}\right\|\right) & \varphi(0) & \cdots & \varphi\left(\left\|B_{1}-B_{N_{b}}\right\|\right) \\
\vdots & \vdots & \vdots & \vdots & \vdots & \vdots \\
\varphi\left(\left\|B_{N_{b}}-I_{1}\right\|\right) & \cdots & \varphi\left(\left\|B_{N_{b}}-I_{N_{i}}\right\|\right) & \varphi\left(\left\|B_{N_{b}}-B_{1}\right\|\right) & \cdots & \varphi(0)
\end{array}\right], \\
\mathbf{U} & =\left[\alpha_{1}^{n}, \alpha_{2}^{n}, \ldots, \alpha_{N_{i}}^{n}, \beta_{1}^{n}, \beta_{2}^{n}, \ldots, \beta_{N_{b}}^{n}\right]^{T}, \\
\mathbf{F} & =\left[f_{1}^{n}\left(I_{1}\right), f_{1}^{n}\left(I_{2}\right), \ldots, f_{1}^{n}\left(I_{N_{i}}\right), \theta_{1}^{n}\left(B_{1}\right), \theta_{1}^{n}\left(B_{2}\right), \ldots, \theta_{1}^{n}\left(B_{N_{b}}\right)\right]^{T} .
\end{aligned}
$$


symmetric. Then we show that it is positive definiteness. For $\forall \xi(\xi \neq 0) \in R^{N_{b}}$,

$$
\begin{aligned}
\left(\mathbf{A}_{1} \xi, \xi\right) & =\sum_{j=1}^{N_{b}} \sum_{k=1}^{N_{b}} \xi_{j} \xi_{k} \varphi_{j}\left(x_{j}^{\prime}\right) \\
& =\sum_{j=1}^{N_{b}} \sum_{k=1}^{N_{b}} \xi_{j} \xi_{k} \varphi\left(\left\|x_{j}-x_{k}\right\|\right) \\
& =\left(\frac{1}{2 \pi}\right)^{N_{b}} \int_{-\infty}^{+\infty} F[\varphi]|\xi(\omega)|^{2} d \omega>0,
\end{aligned}
$$

where $\xi(\omega)=\sum_{j=1}^{N_{b}} \xi_{j} e^{\left.i<\omega, x_{j}\right\rangle}$. So $\mathbf{A}_{1}$ is a symmetric positive definite matrix; that is, $\mathbf{A}_{1}^{-1}$ exists.

Because $\varphi(r)$ is an even function, then $-\varphi(r)$ is an even function too. Moreover, $F[-\varphi]=-(i \omega)^{2} F[\varphi]=\omega^{2} F[\varphi]>$ 0 . Similarly, $\mathbf{A}_{4}$ is also a symmetric positive definite matrix. Hence, $\mathbf{A}_{4}^{-1}$ exits. Now, we have

$$
\left[\begin{array}{cc}
\mathbf{E} & -\mathbf{A}_{2} \mathbf{A}_{4}^{-1} \\
\mathbf{0} & \mathbf{E}
\end{array}\right]\left[\begin{array}{ll}
\mathbf{A}_{1} & \mathbf{A}_{2} \\
\mathbf{A}_{3} & \mathbf{A}_{4}
\end{array}\right]=\left[\begin{array}{cc}
\mathbf{A}_{1}-\mathbf{A}_{2} \mathbf{A}_{4}^{-1} \mathbf{A}_{3} & \mathbf{0} \\
\mathbf{A}_{3} & \mathbf{A}_{4}
\end{array}\right]
$$

Computing the determinants of both sides of (14), we have

$$
|\mathbf{H}|=\left|\begin{array}{ll}
\mathbf{A}_{1} & \mathbf{A}_{2} \\
\mathbf{A}_{3} & \mathbf{A}_{4}
\end{array}\right|=\left|\mathbf{A}_{1}-\mathbf{A}_{2} \mathbf{A}_{4}^{-1} \mathbf{A}_{3}\right| \cdot\left|\mathbf{A}_{4}\right|
$$

As $\left(\mathbf{A}_{3} \mathbf{A}_{1}^{-1} \mathbf{A}_{2}-\mathbf{A}_{4}\right)^{-1}$ exists,

$$
\begin{aligned}
\left(\mathbf{A}_{1}\right. & \left.-\mathbf{A}_{2} \mathbf{A}_{4}^{-1} \mathbf{A}_{3}\right) \\
& \times\left[\mathbf{A}_{1}^{-1}-\mathbf{A}_{1}^{-1} \mathbf{A}_{2}\left(\mathbf{A}_{3} \mathbf{A}_{1}^{-1} \mathbf{A}_{2}-\mathbf{A}_{4}\right)^{-1} \mathbf{A}_{3} \mathbf{A}_{1}^{-1}\right] \\
= & \mathbf{E}-\mathbf{A}_{2}\left(\mathbf{A}_{3} \mathbf{A}_{1}^{-1} \mathbf{A}_{2}-\mathbf{A}_{4}\right)^{-1} \mathbf{A}_{3} \mathbf{A}_{1}^{-1} \\
& -\mathbf{A}_{2} \mathbf{A}_{4}^{-1} \mathbf{A}_{3} \mathbf{A}_{1}^{-1}+\mathbf{A}_{2} \mathbf{A}_{4}^{-1} \mathbf{A}_{3} \mathbf{A}_{1}^{-1} \mathbf{A}_{2} \\
& \times\left(\mathbf{A}_{3} \mathbf{A}_{1}^{-1} \mathbf{A}_{2}-\mathbf{A}_{4}\right)^{-1} \mathbf{A}_{3} \mathbf{A}_{1}^{-1} .
\end{aligned}
$$

In addition,

$$
\begin{aligned}
\mathbf{A}_{2} \mathbf{A}_{4}^{-1} & \mathbf{A}_{3} \mathbf{A}_{1}^{-1} \mathbf{A}_{2}\left(\mathbf{A}_{3} \mathbf{A}_{1}^{-1} \mathbf{A}_{2}-\mathbf{A}_{4}\right)^{-1} \mathbf{A}_{3} \mathbf{A}_{1}^{-1} \\
& -\mathbf{A}_{2}\left(\mathbf{A}_{3} \mathbf{A}_{1}^{-1} \mathbf{A}_{2}-\mathbf{A}_{4}\right)^{-1} \mathbf{A}_{3} \mathbf{A}_{1}^{-1} \\
= & \mathbf{A}_{2} \mathbf{A}_{4}^{-1}\left(\mathbf{A}_{3} \mathbf{A}_{1}^{-1} \mathbf{A}_{2}-\mathbf{A}_{4}\right) \\
& \times\left(\mathbf{A}_{3} \mathbf{A}_{1}^{-1} \mathbf{A}_{2}-\mathbf{A}_{4}\right)^{-1} \mathbf{A}_{3} \mathbf{A}_{1}^{-1} \\
= & \mathbf{A}_{2} \mathbf{A}_{4}^{-1} \mathbf{A}_{3} \mathbf{A}_{1}^{-1} .
\end{aligned}
$$

By (16) and (17), we obtain

$$
\begin{aligned}
& \left(\mathbf{A}_{1}-\mathbf{A}_{2} \mathbf{A}_{4}^{-1} \mathbf{A}_{3}\right) \\
& \quad \times\left[\mathbf{A}_{1}^{-1}-\mathbf{A}_{1}^{-1} \mathbf{A}_{2}\left(\mathbf{A}_{3} \mathbf{A}_{1}^{-1} \mathbf{A}_{2}-\mathbf{A}_{4}\right)^{-1} \mathbf{A}_{3} \mathbf{A}_{1}^{-1}\right]=\mathbf{E} .
\end{aligned}
$$

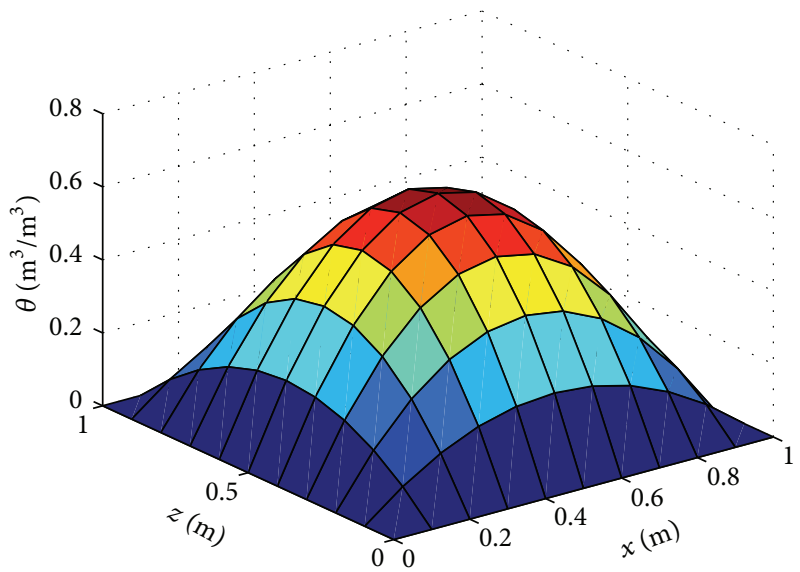

FIGURE 2: The exact solutions of Example 1.

So

$$
\begin{aligned}
& \left(\mathbf{A}_{1}-\mathbf{A}_{2} \mathbf{A}_{4}^{-1} \mathbf{A}_{3}\right)^{-1} \\
& =\mathbf{A}_{1}^{-1}-\mathbf{A}_{1}^{-1} \mathbf{A}_{2}\left(\mathbf{A}_{3} \mathbf{A}_{1}^{-1} \mathbf{A}_{2}-\mathbf{A}_{4}\right)^{-1} \mathbf{A}_{3} \mathbf{A}_{1}^{-1} .
\end{aligned}
$$

According to (15) and (19), we know that $|\mathbf{H}| \neq \mathbf{0}$. Thus, the matrix equation (10) has a unique solution. Now the numerical solution to problem (1), based on the radial basis function collocation method, can be calculated.

\section{Numerical Examples}

Example 1. Solve the following linear model using the radial basis function collocation method:

$$
\begin{gathered}
\frac{\partial \theta}{\partial t}=\frac{\partial}{\partial x}\left(D(\theta) \frac{\partial \theta}{\partial x}\right)+\frac{\partial}{\partial z}\left(D(\theta) \frac{\partial \theta}{\partial z}\right)+f, \\
(x, z) \in \Omega=[0,1] \times[0,1], \\
\theta(x, z, 0)=\theta_{0}, \quad(x, z) \in \Omega, \\
\theta(x, z, t)=\theta_{1}, \quad(x, z) \in \partial \Omega, t>0 .
\end{gathered}
$$

Let $D(\theta)=1$; then the analytic solution of $(20)$ is $\theta(z, t)=$ $\left(x^{2}-x\right)\left(z^{2}-z\right) t$ and $f=\left(x^{2}-x\right)\left(z^{2}-z\right)-2 t\left(x^{2}+z^{2}-x-z\right)$. The values of $\theta_{0}$ and $\theta_{1}$ are determined by analytic solution using a spatial step $h=0.1$ and a time step $\Delta t=0.01$ from $t=0$ to $t=10$. The Gaussian function $\exp \left(-c r^{2}\right)$ is selected as the radial basis function and the error estimate is based on $L_{2}$-norm. The numerical and exact solutions of Example 1 are shown in Figures 2 and 3, respectively.

Table 1 shows a comparison of this method with FEM, where $h=0.1$ and $t=10$.

Next, we consider the time step $\Delta t=0.01$, a different spatial step, and different parameters $c$ in the Gaussian function $\exp \left(-c r^{2}\right)$. The results are shown in Table 2 .

For the linear model, Figures 1-4 and Table 1 show that the method presented in this paper is feasible. And comparing it with the traditional method, it showed good accuracy and rapid convergence rate. And Table 2 shows that 
TABLE 1: The results of the new method compared with FEM.

\begin{tabular}{lcccc}
\hline Numerical methods & $t$ & Calculation errors & Calculation time(s) & Degree of convergence \\
\hline \multirow{2}{*}{ New method } & 0.5 & $8.4197 e-004$ & 1.230020 & 2.7300 \\
& 1.0 & $9.1056 e-004$ & 2.427944 & 2.4725 \\
\hline \multirow{2}{*}{ FEM } & 0.5 & $1.0017 e-003$ & 1.021520 & 2.6720 \\
& 1.0 & $1.6667 e-003$ & 3.825802 & 2.2707 \\
\hline
\end{tabular}

TABLE 2: Numerical solutions of Example 1 related to the parameter and spatial step.

\begin{tabular}{cccc}
\hline Spatial step & Parameters $c$ & Calculation errors & Calculation time(s) \\
\hline \multirow{3}{*}{0.1} & 9.0 & $3.4000 e-003$ & 1.372784 \\
& 9.5 & $9.1056 e-004$ & 2.427944 \\
& 9.6 & $3.4000 e-003$ & 1.580791 \\
0.25 & 0.33 & $1.1000 e-003$ & 0.672394 \\
& 0.35 & $5.7098 e-004$ & 0.659509 \\
& 0.4 & $9.2511 e-004$ & 0.659885 \\
\hline
\end{tabular}

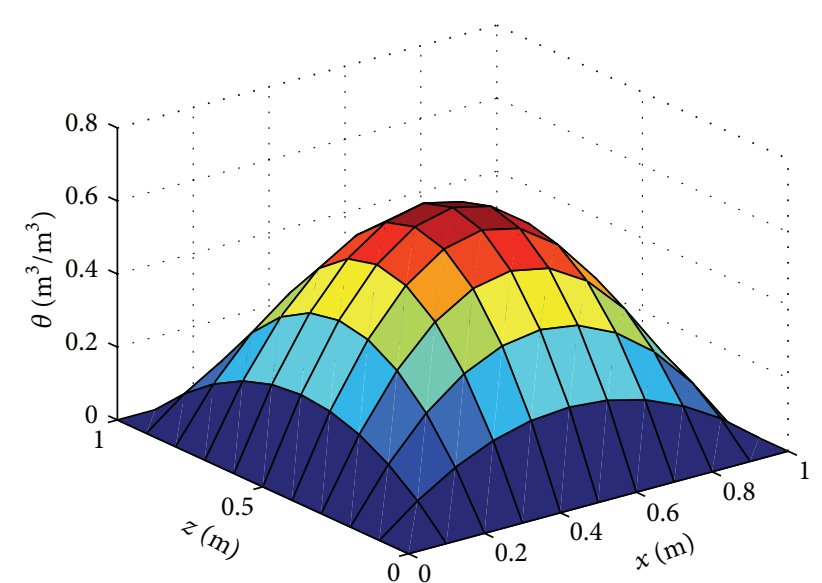

Figure 3: Numerical solutions using the radial basis function collocation method.

the computational accuracy and computing time are both related to the selections of parameter $c$ in the radial basis functions and the spatial step.

Example 2. We consider the 2D horizontal absorption soil water movement equation:

$$
\begin{gathered}
\frac{\partial \theta}{\partial t}=\frac{\partial}{\partial x}\left(D(\theta) \frac{\partial \theta}{\partial x}\right)+\frac{\partial}{\partial z}\left(D(\theta) \frac{\partial \theta}{\partial z}\right)+f \\
(x, z) \in \Omega=[0,1] \times[0,1], \\
\theta(x, z, 0)=\theta_{0}, \quad(x, z) \in \Omega, \\
\theta(x, z, t)=\theta_{1}, \quad(x, z) \in \partial \Omega, t>0 .
\end{gathered}
$$

Here $D(\theta)=0.01 e^{\theta}, f=-0.02 t^{2}\left(x-x^{2}\right)\left(z-z^{2}\right)\left(x^{2}-x+\right.$ $\left.z^{2}-z\right)-0.01 t^{2}\left(z^{2}(2 x z-z-2 x+1)^{2}\right)+x^{2}(2 x z-2 z-x+$ $1)^{2}+\left(x-x^{2}\right)\left(z^{2}-z\right)$ and the analytic solution is $\theta(z, t)=\left(x^{2}-\right.$ $x)\left(z^{2}-z\right) t$. The values of $\theta_{0}$ and $\theta_{1}$ are determined by analytic solution. It is a nonlinear equation with the spatial step

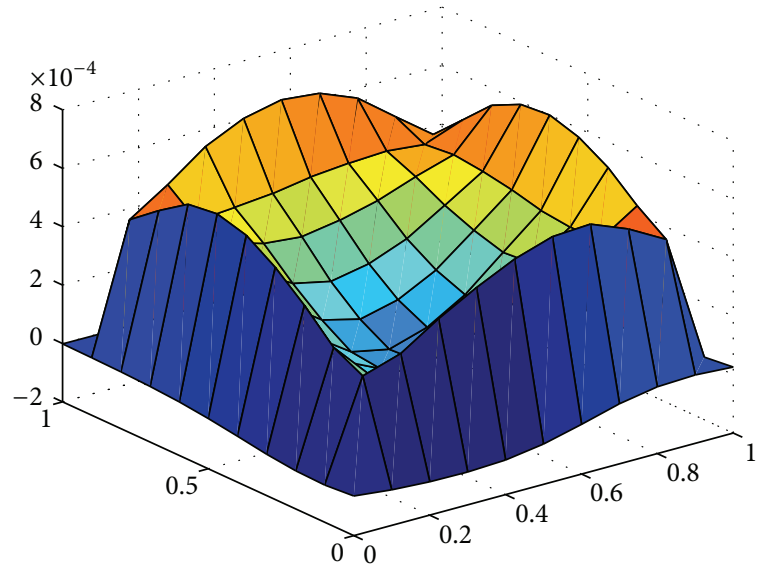

FIGURE 4: The error graph of the new method.

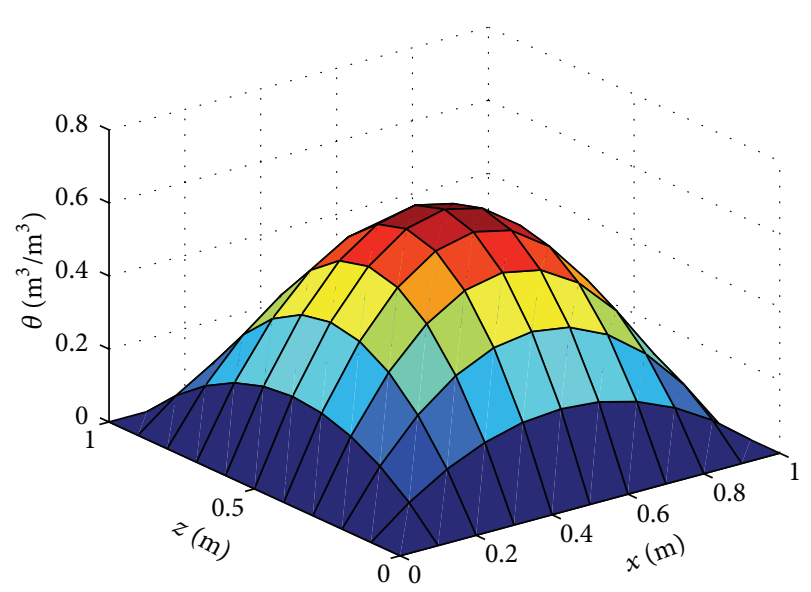

FIgURE 5: The exact solutions of Example 2.

$h=0.1$ and time step $\Delta t=0.01$ from $t=0$ to $t=$ 10. The radial basis function is also a Gaussian function $\exp \left(-c r^{2}\right)(c>0)$. The error estimate is based on $L_{2}$-norm. 
TABLE 3: The results of the new method compared with FEM for Example 2.

\begin{tabular}{lcccc}
\hline Numerical methods & $t$ & Calculation errors & Calculation time(s) & Degree of convergence \\
\hline \multirow{2}{*}{ New method } & 0.5 & $4.6462 e-005$ & 2.100832 & 3.6871 \\
& 1.0 & $9.6914 e-005$ & 3.782251 & 3.2203 \\
\hline \multirow{2}{*}{ FEM } & 0.5 & $1.0701 e-004$ & 2.201250 & 3.4186 \\
& 1.0 & $1.5367 e-004$ & 3.882052 & 3.0664 \\
\hline
\end{tabular}

TABLE 4: Numerical solution based on different spatial steps and different parameters in Example 2.

\begin{tabular}{lccc}
\hline Spatial step & Parameters $c$ & Calculation errors & Calculation time(s) \\
\hline \multirow{2}{*}{0.1} & 10 & $3.1488 e-004$ & 2.995965 \\
& 11 & $9.6914 e-005$ & 3.782251 \\
\hline \multirow{2}{*}{0.25} & 12 & $1.0218 e-004$ & 4.800045 \\
& 0.45 & $1.5427 e-004$ & 1.682164 \\
& 0.50 & $7.7579 e-005$ & 1.645171 \\
& 0.55 & $9.8661 e-005$ & 1.686100
\end{tabular}

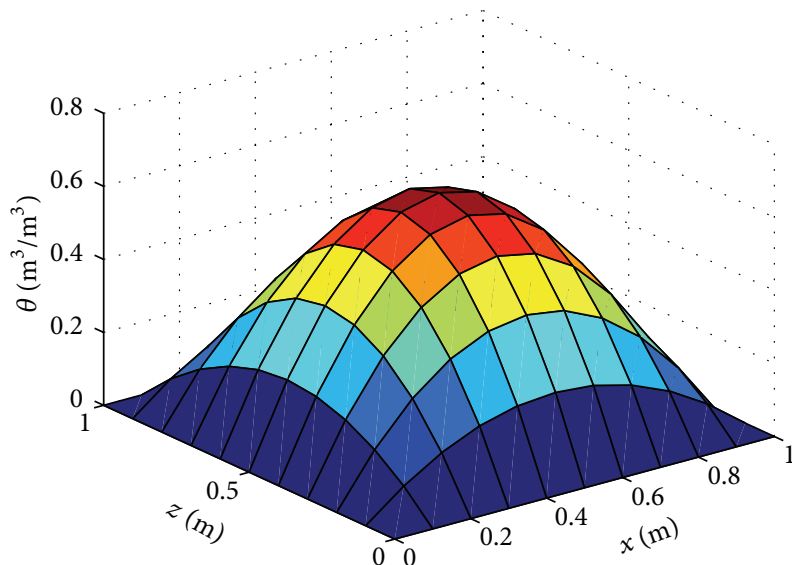

Figure 6: Numerical solutions using the radial basis function collocation method.

The numerical and exact solutions of Example 2, along with the error, are shown in Figures 5, 6, and 7, respectively.

Table 3 shows the comparison of this method with FEM, where $h=0.1$ and $t=10$

For the time step $\Delta t=0.01$, we give the results of the new method based on different spatial steps and different parameters $c$. These are shown in Table 4 .

For the nonlinear soil water movement equation, we can get the same conclusions as in linear equation.

\section{Conclusions}

In this paper, a mathematical model with the boundary conditions for soil water movement under irrigation has been developed by constructing a radial basis function collocation method. The existence and uniqueness of the solution were proven. Several numerical examples show that the proposed method yields higher precision and is easier to solve $2 \mathrm{D}$ soil

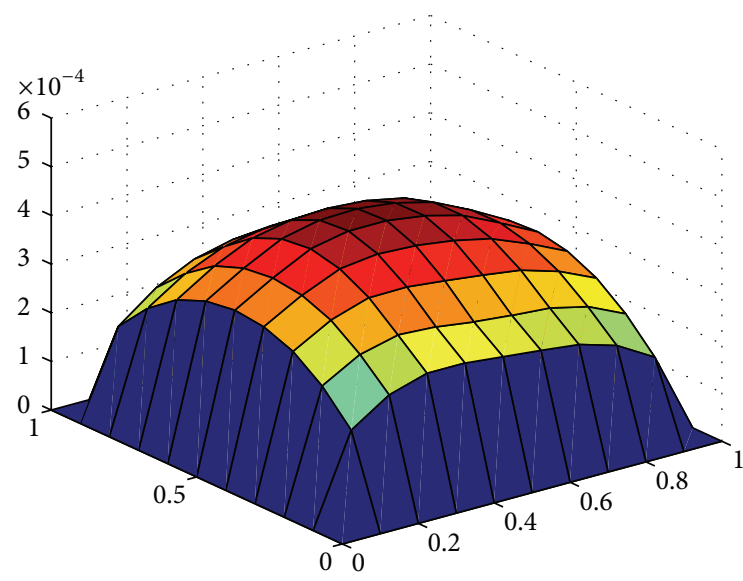

FIGURE 7: The error graph.

water movement equations than traditional methods. Moreover, the selection of the time, spatial steps, and parameter $c$ has a direct influence on calculation accuracy. Therefore, it is necessary to study the combination of steps, radial basis function, and parameter $c$ to obtain the numerical solutions. In addition, it lays the foundation for the numerical solutions to high-dimensional soil water movement equations, which is very important.

\section{Conflict of Interests}

The authors declare that there is no conflict of interests regarding the publication of this paper.

\section{Acknowledgments}

This paper is supported by National Natural Science Foundation of China (NSFC 11101330, 51305344) and Scientific Foundation of Shaanxi Province (2014K05-22). 


\section{References}

[1] L. B. Lucyt, "A numerical approach to the testing of the fission hypothesis," The Astronomical Journal, vol. 82, pp. 1013-1024, 1977.

[2] R. A. Gingold and J. J. Monaghan, "Smoothed particle hydrodynamics: theory and application to non-spherical stars," Monthly Notices of the Royal Astronomical Society, vol. 181, pp. 375-389, 1977.

[3] B. Nayroles, G. Touzot, and P. Villon, "Generalizing the finite element method: diffuse approximation and diffuse elements," Computational Mechanics, vol. 10, no. 5, pp. 307-318, 1992.

[4] T. Belytschko, Y. Y. Lu, and L. Gu, "Element-free Galerkin methods," International Journal for Numerical Methods in Engineering, vol. 37, no. 2, pp. 229-256, 1994.

[5] R. Schaback, "Improved error bounds for scattered data interpolation by radial basis functions," Mathematics of Computation, vol. 68, no. 225, pp. 201-216, 1999.

[6] N. Sukumar, B. Moran, and T. Belytschko, "The natural element method in solid mechanics," International Journal for Numerical Methods in Engineering, vol. 43, no. 5, pp. 839-887, 1998.

[7] Z. Wu, "About the convergence of interpolation with radial basis function," Annals of Mathematics, vol. 14A, pp. 480-486, 1993.

[8] H. Wendland, "Piecewise polynomial, positive definite and compactly supported radial functions of minimal degree," Advances in Computational Mathematics, vol. 4, no. 1, pp. 389396, 1995.

[9] H. Wendland, "Meshless Galerkin methods using radial basis functions," Mathematics of Computation, vol. 68, no. 228, pp. 1521-1531, 1999.

[10] S. N. Atluri and S. Shen, The Meshless Local Petrov-Galerkin (MLPG) Method, Tech Science Press, Encino, Calif, USA, 2002.

[11] G. R. Liu, Meshfree Methods: Moving beyond the Finite Element Method, CRC Press, Boca Raton, Fla, USA, 2002.

[12] W. K. Liu, Y. Chen, R. A. Uras, and C. T. Chang, "Generalized multiple scale reproducing kernel particle methods," Computer Methods in Applied Mechanics and Engineering, vol. 139, no. 1-4, pp. 91-157, 1996.

[13] C. A. Duarte and J. T. Oden, "An $\$$ h\$-\$p\$ adaptive method using clouds," Computer Methods in Applied Mechanics and Engineering, vol. 139, no. 1-4, pp. 237-262, 1996.

[14] I. Babuška and J. M. Melenk, "The partition of unity method," International Journal for Numerical Methods in Engineering, vol. 40, no. 4, pp. 727-758, 1997.

[15] E. Oñate, S. Idelsohn, O. C. Zienkiewicz, and R. L. Taylor, "A finite point method in computational mechanics: applications to convective transport and fluid flow," International Journal for Numerical Methods in Engineering, vol. 39, no. 22, pp. 38393866, 1996.

[16] N. R. Aluru, "A point collocation method based on reproducing kernel approximations," International Journal for Numerical Methods in Engineering, vol. 47, no. 6, pp. 1083-1121, 2000.

[17] T. J. Liszka, C. Duarte, and W. W. Tworzydlo, "Hp-meshless cloud method," Computer Methods in Applied Mechanics and Engineering, vol. 139, no. 1-4, pp. 263-288, 1996.

[18] T. Zhu, J. Zhang, and S. N. Atluri, "A local boundary integral equation (LBIE) method in computational mechanics, and a meshless discretization approach," Computational Mechanics, vol. 21, no. 3, pp. 223-235, 1998.

[19] X. Zhang and Y. Liu, Meshless Methods, Tsinghua University Press, Beijing, China, 2004.
[20] E. J. Kansa, "Multiquadrics—a scattered data approximation scheme with applications to computational fluid-dynamics. I. Surface approximations and partial derivative estimates," Computers \& Mathematics with Applications, vol. 19, no. 8-9, pp. 127-145, 1990.

[21] G. E. Fasshauer, "Solving differential equations with radial basis functions: multilevel methods and smoothing," Advances in Computational Mathematics, vol. 11, no. 2-3, pp. 139-159, 1999.

[22] Y. C. Hon, K. F. Cheung, X. Z. Mao, and E. J. Kansa, "Multiquadric solution for shallow water equations," Journal of Hydraulic Engineering, vol. 125, no. 5, pp. 524-533, 1999.

[23] S. Rippa, "An algorithm for selecting a good value for the parameter $c$ in radial basis function interpolation," Advances in Computational Mathematics, vol. 11, no. 2-3, pp. 193-210, 1999.

[24] M. D. Buhmann, "A new class of radial basis functions with compact support," Mathematics of Computation, vol. 70, no. 233, pp. 307-318, 2001.

[25] L. W. Zhang, Y. J. Deng, and K. M. Liew, "An improved elementfree Galerkin method for numerical modeling of the biological population problems," Engineering Analysis with Boundary Elements, vol. 40, pp. 181-188, 2014.

[26] R. J. Cheng, L. W. Zhang, and K. M. Liew, "Modeling of biological population problems using the element-free kp-Ritz method," Applied Mathematics and Computation, vol. 227, pp. 274-290, 2014.

[27] P. F. Guo, L. W. Zhang, and K. M. Liew, "Numerical analysis of generalized regularized long wave equation using the elementfree kp-Ritz method," Applied Mathematics and Computation, vol. 240, pp. 91-101, 2014.

[28] J. W. Yan, L. W. Zhang, K. M. Liew, and L. H. He, "A higher-order gradient theory for modeling of the vibration behavior of singlewall carbon nanocones," Applied Mathematical Modelling, vol. 38, no. 11-12, pp. 2946-2960, 2014.

[29] Z. X. Lei, L. W. Zhang, K. M. Liew, and J. L. Yu, "analysis of carbon nanotube-reinforced functionally graded cylindrical panels using the element-free kp-Ritz method," Composite Structure, vol. 113, pp. 328-338, 2014.

[30] X. F. Pan, X. Zhang, and M. W. Lu, "Meshless Galerkin leastsquares method," Computational Mechanics, vol. 35, no. 3, pp. 182-189, 2005.

[31] Y. Gu and L. Zhang, "Coupling of the meshfree and finite element methods for determination of the crack tip fields," Engineering Fracture Mechanics, vol. 75, pp. 986-1004, 2008.

[32] Y. Duan and Y. Tan, "On condition number of meshless collocation method using radial basis functions," Applied Mathematics and Computation, vol. 172, no. 1, pp. 141-147, 2006.

[33] H. Hu, Z. Li, and A. H. Cheng, "Radial basis collocation methods for elliptic boundary value problems," Computers and Mathematics with Applications, vol. 50, no. 1-2, pp. 289-320, 2005.

[34] L. Su, X. Qin, B. Miao, and Q. Wang, "Radial basis function collocation method with difference for nonlinear convectiondominated diffusion equations," in Proceedings of the 6th International Conference on Natural Computation (ICNC '10), pp. 3203-3207, Yantai, China, August 2010.

[35] W. Guo, B. Li, Z. Ji, Y. Jiang, and F. Yan, “Two-dimensional numerical simulation of soil water infiltration under bedirrigating sowing," Transactions of the Chinese Society of Agricultural Engineering, vol. 17, no. 2, pp. 24-27, 2001.

[36] S. P. Neuman, "Saturated-unsaturated seepage by finite elements," Journal of the Hydraulics Division, vol. 99, no. 12, pp. 2233-2250, 1973. 
[37] H. Zhang and S. Chen, "Numerical simulation of infiltration in unsaturated soil," Rock and Soil Mechanics, vol. 24, no. 5, pp. 715-718, 2003.

[38] X. Lu and D. H. Wu, "Numerical simulation of infiltration in unsaturated soil," China Water Transport, vol. 4, no. 4, pp. 136137, 2006.

[39] H. R. Li and Z. D. Luo, "Semi-discrete finite volume element simulation for two-dimensional unsaturated soil water flow problem," Mathematica Numerica Sinica, vol. 33, no. 1, pp. 5768, 2011.

[40] D. Zhou and H. Wang, "Application of RBF in simulation of groundwater flow," Journal of Liaoning Normal University, vol. 31, no. 4, pp. 390-392, 2008. 


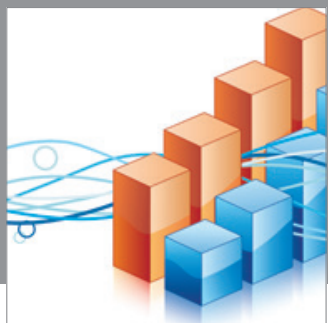

Advances in

Operations Research

mansans

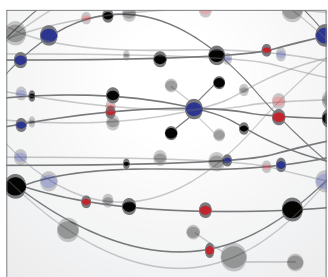

The Scientific World Journal
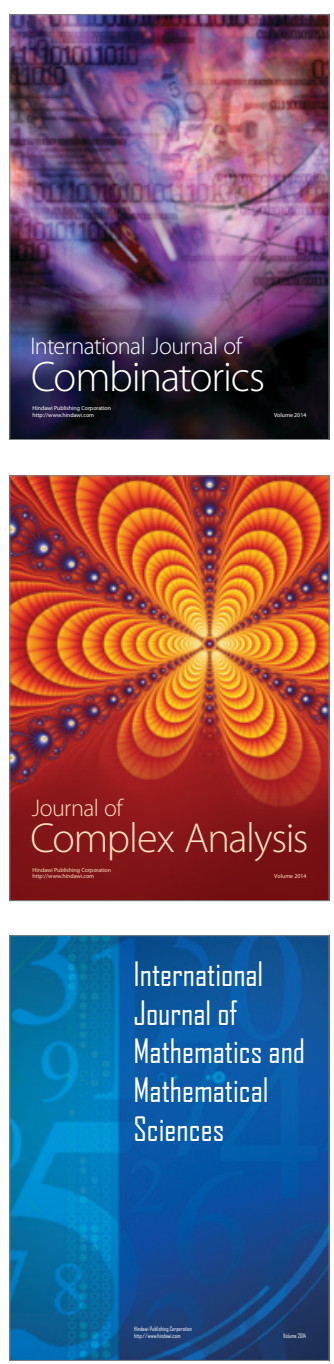
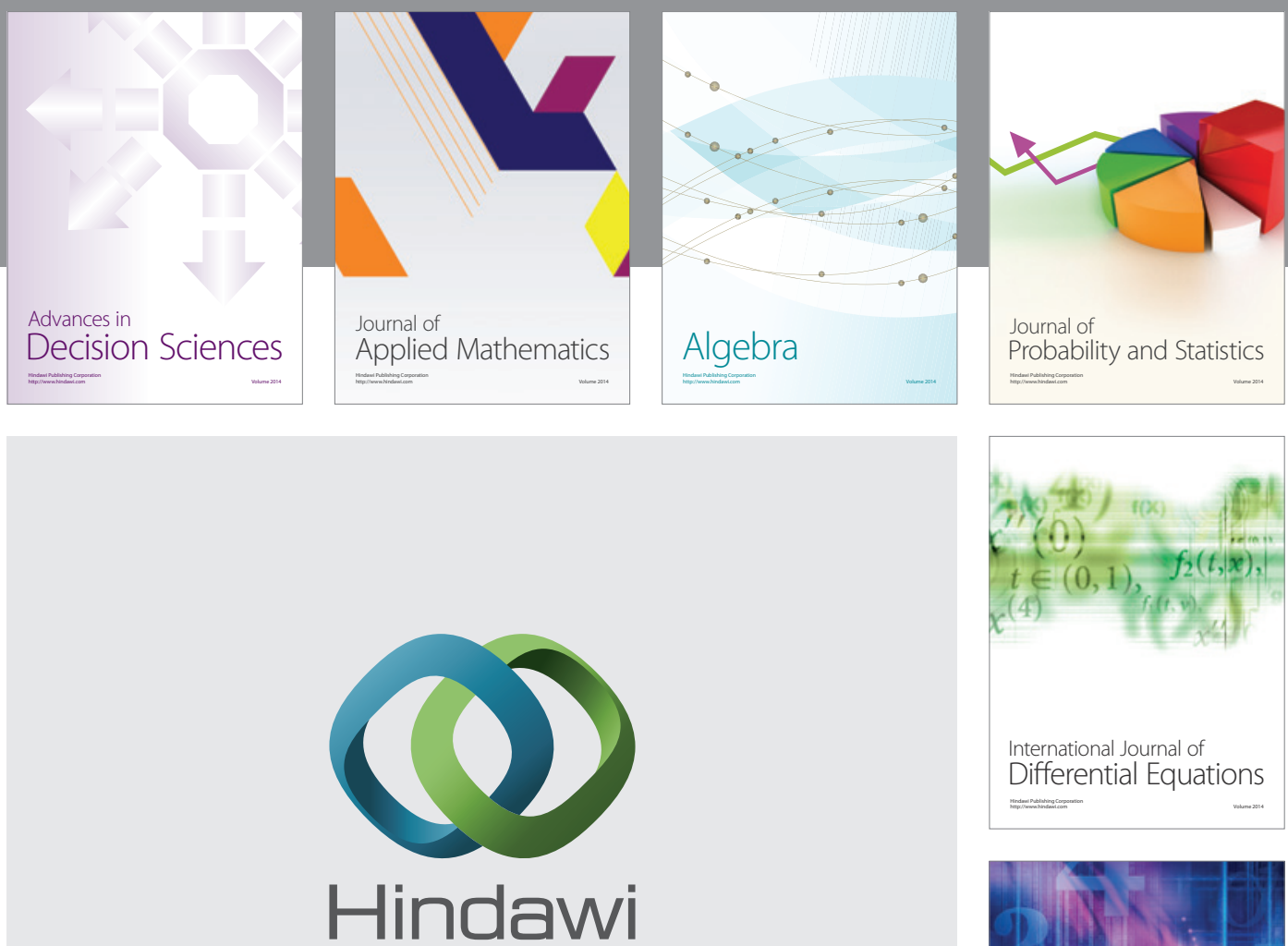

Submit your manuscripts at http://www.hindawi.com
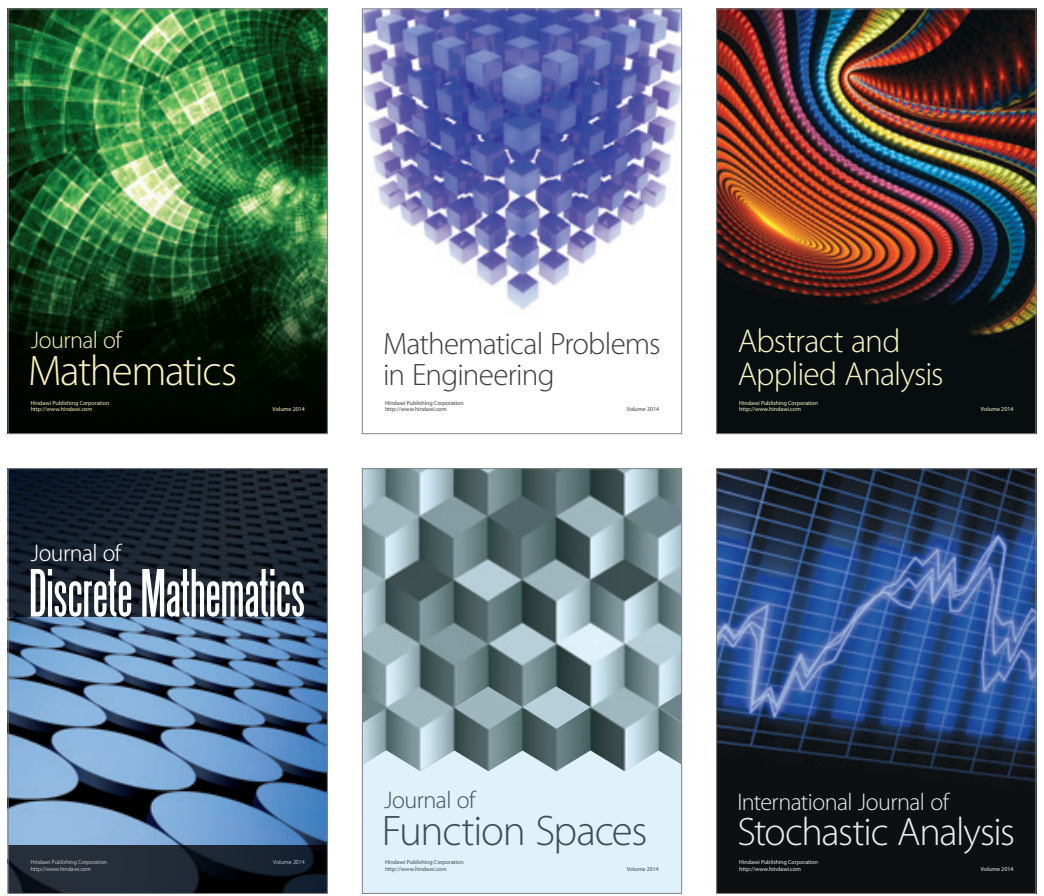

Journal of

Function Spaces

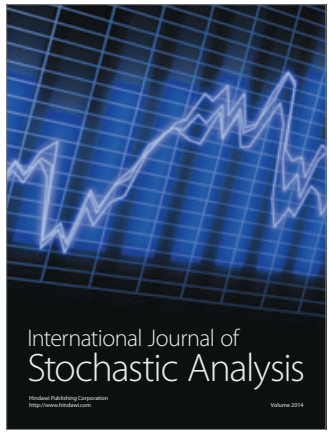

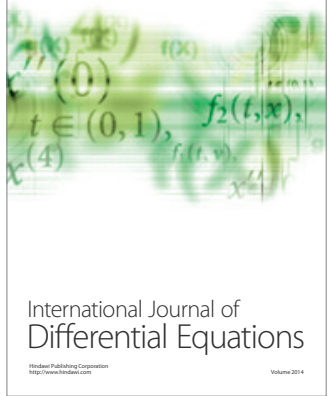
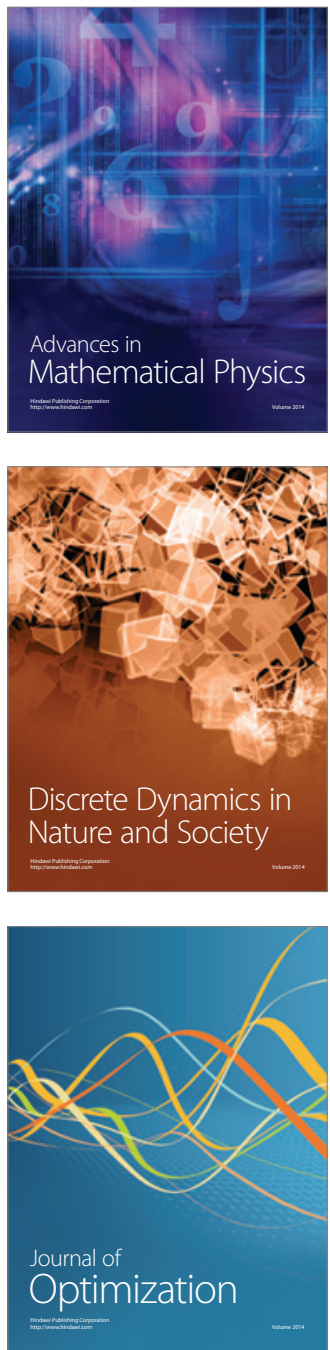